\title{
FANET optimization: a destination path flow model
}

\author{
B. O. Sadiq, A. T. Salawudeen
}

Department of Computer Engineering, Ahmadu Bello University Zaria, Nigeria

\begin{tabular}{l} 
Article Info \\
\hline Article history: \\
Received Dec 14, 2019 \\
Revised Feb 29, 2020 \\
Accepted Mar 8, 2020 \\
\hline
\end{tabular}

Keywords:

Destination path model FANET optimization FANET routing protocol Media and sport coverage Smell agent optimization

\begin{abstract}
Closed-loop routing in flying ad hoc networks (FANET) arises as a result of the quick changes of communication links and topology. As such, causing link breakage during information dissemination. This paper proposed a destination path flow model to improve the communication link in FANET. The models utilized Smell Agent Optimization and Particle Swarm Optimization algorithms in managing link establishment between communicating nodes. The modeled scenario depicts the practical application of FANET in media and sports coverage where only one vendor is given the license for live coverage and must relay to other vendors. Three different scenarios using both optimization Algorithms were presented. From the result obtained, the SAO optimizes the bandwidth costs much better than PSO with a percentage improvement of $10.46 \%, 4.04 \%$ and $3.66 \%$ with respect to the 1 st, 2 nd and 3rd scenarios respectively. In the case of communication delay between the FANET nodes, the PSO has a much better communication delay over SAO with percentage improvement of $40.89 \%, 50.26 \%$ and $68.85 \%$ in the first, second and third scenarios respectively.
\end{abstract}

Copyright $\odot 2020$ Institute of Advanced Engineering and Science. All rights reserved.

\section{Corresponding Author:}

B. O. Sadiq,

Department of Computer Engineering,

Ahmadu Bello University,

P.O Box 1044, Kaduna State, Nigeria.

Email: bosadiq@abu.edu.ng

\section{INTRODUCTION}

flying ad hoc network (FANET) is a type of ad hoc network in which Small unmanned aerial vehicles (UAV) constitute its nodes [1]. Due to UAVs widening performance, it has been employed not only in military functions but also in civilian activities like media coverage, traffic monitoring, rescue operation and agriculture [2-5]. Nodes in FANET are connected in an ad hoc manner for mission fulfillment without necessarily needing an infrastructure. Mission fulfillment in FANET is highly dependent on the routing algorithms [6-7]. FANET routing algorithms are either centralized or distributed. In centralized routing algorithms, a computer or node can be fed with the full network knowledge required to solve the problem while in distributed algorithms it is sometimes not possible to communicate with the central computer or node [8]. Hence, making nodes communicate with one another. When nodes communicate with one another, it is important to efficiently manage the links in order to prevent a closed routing loop [9]. In the situation whereby, the routing loop is closed, traffic entering a node never gets to its destination. Hence, saturating the link capacity [10]. FANET usage in civilian applications increased the need of having a reliable connection amongst the nodes and control centers. Nonetheless, this is affected by frequent topology change and the operation environment. This can cause the frequent breakage of network links between the UAVs and control centers [11]. FANET often uses connected network topology as depicted in Figure 1. 


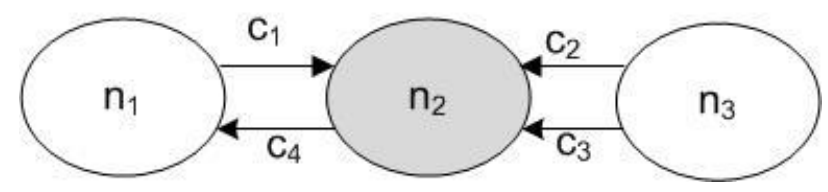

Figure 1. FANET connected network topology

Routing problems in ad hoc networks can be generalized as either a flow-based routing or a distributed-based routing. Nodes in the flow-based routing identify the demand $(\mathrm{kx})$ of the arriving traffic and make a different per-demand routing decision. This is why they are called connection-oriented routing. During this process, the network determines signals flowing through a route and updates the routing table of the traversed nodes accordingly. Based on this, the flow data frames are attached with enough control information in their headers with a view to enforcing the previously defined route in the intermediate nodes. On the contrary to the flow-based routing, the distributed-based routing is connectionless oriented where by a source node can inject traffic to the network without any prior connection establishment. However, both routing problems in FANET are affected by its inherent characteristics such as the fast and random nature of moving nodes and topological changes [12-16].

Motivated by these challenges, we focused attention on the use of optimization techniques [17-21] to develop a path flow model that will reduce link breakage generated in the FANET environment by finding practical solutions. From online sister algorithms, we are interested in preventing closed-loop routing that negatively affects link capacity and efficiency as well as causing link breakage in FANET. Moved by realistic observations in the use of FANET, this paper proposed a model for destination-link, flow-path and flow-link problems in UAV networks with application in the sport and media coverage. As such, the main contributions are as follows:

- We designed and implemented a realistic path model for information dissemination in FANET using smell agent optimization and Particle Swarm Optimization.

- Based on the design in (i), we proposed a suitable and efficient FANET routing methodology.

The remaining aspect of the paper is itemized as follows: section two presents the review of related works, its contributions, and limitations. In section three, the model formulation, as well as governing equations, are presented. Results analysis and conclusions drawn from the results are presented in section four and section five respectively.

\section{RELATED WORKS}

A number of works in literature have modeled link availability in ad hoc networks relating to mobile ad hoc network (MANET) or vehicular ad hoc network (VANET) [21-24]. However, the inherent characteristics of FANET make the models unsuitable. One of the major problems faced by FANET is the nature of the fast-moving nodes and dynamic topology change. A solution to this problem is the design of a FANET protocol that will concentrate on these inherent characteristics [2, 4]. The authors in [2] were one of the earliest to design a specific protocol for FANET. The protocol took into consideration the inherent characteristics of FANET. However, the path to the destination was not optimized which might lead to a close-loop routing. The authors in the work of [4] developed a novel predictive routing strategy in FANET with a view to minimizing link breakage due to the nature of nodes. The authors combined the use of both directional and omnidirectional antenna to improve routing path using derived expressions which are the expected connection time (EMC) and Utility function for path selection. However, their approach did not optimize the routing path which could still lead to a closed-loop routing.

A modification of the existing MANET routing protocol for FANET applications was presented by $[11,14]$. Nonetheless, the protocols were unsuitable for FANET because they maintained routing information that might never be used. Thus, leading to a large amount of routing overhead that causes information loss. [25-30] are some of the authors that concentrated on improving the communication models in FANET. Muhammad et al., [25] and Antinio et al., [26] developed a hybrid communication scheme for the efficient and low-cost deployment of future FANET and a new domain for communications in FANET respectively. The architecture in [25] combined the use of short-range wireless communication technology which is the Bluetooth and wide range communication technology which is the cellular network. This was done with a view to improving communication efficiency. However, both works did not consider routing path and link availability. The work of [27] addresses the high mobility in FANET by means of a clustering algorithm. A communication model was developed and benchmarked against artificial 
intelligence techniques such as Ant Colony Optimization-based clustering algorithm and Greywolf Optimization-based clustering algorithm. Nonetheless, their work focused on saving Nodes' energy by means of controlling their transmission range and efficiently clustering the network and not a path model to improve routing efficiency. In order to solve scenario-based problems in FANET, the authors in the work of [28] presented FANET applications scenario and models. The research article listed mobility models and provided guidance in understanding whether the models could be adopted depending on the specific flying ad-hoc network application scenarios. Based on the reviewed models in this article, this research work will adopt the random mobility model with collision anticipation. In view of the reviewed works and with respect to the state-of-the-art approach in the literature, there has been limited work in literature to improve network connectivity in FANET through path optimization. As such, this work presented an optimized destination path model that will improve routing efficiency in FANET.

\section{PROPOSED LINK CONNECTIVITY FORMULATION}

The formulation of the path model are as follows

\subsection{Basic assumption and problem input}

There are basically three types of FANET nodes: Source nodes, intermediate nodes, and destination nodes. Source nodes generate traffic or data to be sent. Intermediate nodes act as a relay node between the source node and the destination nodes. The destination nodes are the recipient of the generated traffic or data. Each node is equipped with a camera for video data capture. The location of nodes is assumed to be available to the control system all the time with the aid of a GPS. Each node in the network is assumed to have a certain wireless connection range and a maximum velocity. Given a network topology, G(N, C), two nodes in the network $n_{1,} n_{2} \in N$ at positions $\left\{r_{x t} s_{x t}\right\}$ are at a distance apart:

$$
d_{x y t}=\sqrt{\sum_{i=1}^{n}\left(r_{i}-s_{i}\right)^{2}}
$$

The communication strength between two nodes is dependent on the distance between them. Following other academic [29] and industrial [30] papers, normalized transmission rates and ranges are adopted in this work. With application to sports coverage, we adopted a football field scenario of 160 feet $(48.5 \mathrm{~m})$ wide and 360 feet $(109.1 \mathrm{~m})$ long. Works of literature have also shown that the standard speed of a FANET node is between $10 \mathrm{~m} / \mathrm{s}$ to $500 \mathrm{~m} / \mathrm{s}$ depending on the manufacturer's specification. But in this work, we used $10 \mathrm{~m} / \mathrm{s}$ as the speed of the FANET nodes. We assumed that only one of the FANET node is given the license to cover the sport or media event. As such, the node must do a multicast to other FANET nodes of different vendors as shown in Figure 2.

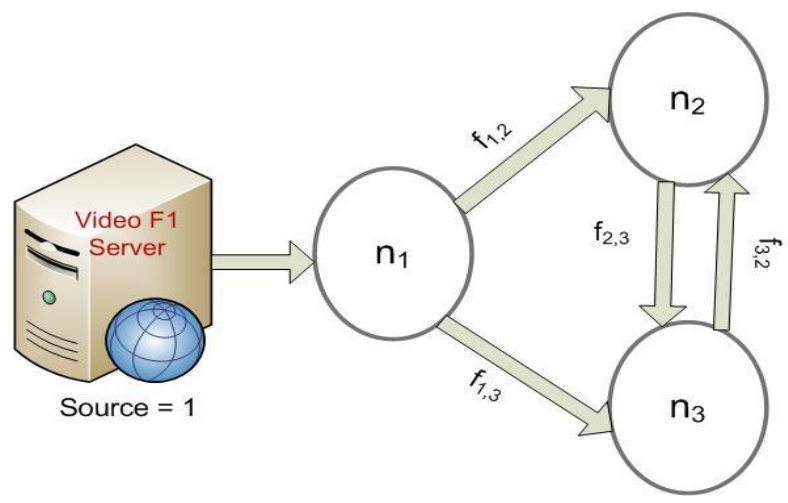

Figure 2. FANET multicast scenario

In order to solve the routing problem, we define a variable that specifies the route through which the flow of information will be transmitted. A variable for information transmission is defined as follows:

$$
M_{z}^{t f}= \begin{cases}1 & \text { if link (x,y) is used for flow } \mathrm{f} \text { with destination } \mathrm{t} \\ 0 & \text { if otherwise }\end{cases}
$$


This means that the variable vector $M_{x y}^{t f}$ is 1 if the links (x,y) is used to transmit flow $\mathrm{f}$ with destination $\mathrm{t}$ and 0 if a link $(\mathrm{x}, \mathrm{y})$ is not used for flow $\mathrm{f}$ with destination $\mathrm{t}$. Table 1 presents the parameters that will be used for FANET in the multicast scenario.

Table 1. FANET parameters for multicast scenario

\begin{tabular}{ll}
\hline FANET Terms & Definition \\
\hline $\mathrm{G}(\mathrm{N}, \mathrm{C})$ & Graphs of the topology \\
$\mathrm{N}$ & Set of nodes \\
$\mathrm{C}$ & Set of links \\
$(\mathrm{x}, \mathrm{y})$ & Link from node x to node $\mathrm{y}$ \\
$\mathrm{F}$ & Flow set \\
$\mathrm{P}$ & Path \\
$\mathrm{f}$ & Any multicast flow \\
$\mathrm{d}_{\mathrm{xyt}}$ & Distance between nodes with time \\
$\mathrm{k}_{\mathrm{xf}}$ & Traffic demand for flow f \\
$\mathrm{b}_{\mathrm{xy}}$ & The available capacity of each link (x,y) \\
$M_{z}^{t f}$ & Indicate whether the link $(\mathrm{x}, \mathrm{y})$ is used for flow f \\
\hline
\end{tabular}

\subsection{Information flow formulation}

Consider the multicast scenario given in Figure 2, assuming the information flow between the links is represented as $f$, then the link capacity of every interlinked node can be computed. For example, link $d_{12}$ which connect node $n_{1}$ and $n_{2}$ are:

$$
f_{1,2}=\left\|n_{1}-n_{2}\right\|
$$

Similarly, the information flow through the other links can be expressed as follows:

$$
\begin{aligned}
& f_{1,3}=\left\|n_{1}-n_{3}\right\| \\
& f_{2,3}=\left\|n_{2}-n_{3}\right\| \\
& f_{3,2}=\left\|n_{3}-n_{2}\right\|
\end{aligned}
$$

where \|\| is the Euclidean norm or the $L_{2}$-normal between the FANET nodes. From the FANET multicast scenario of Figure 2, node $n_{1}$ relay information bandwidth received from the video server. Node $n_{1}$ then transmits information to $n_{2}$ and $n_{3}$. When only one of $n_{2}$ or $n_{3}$ receives information from $n_{1}$, the other node can connect indirectly through either of $n_{2}$ or $n_{3}$. For a complete information flow between the three FANET nodes, three scenarios of connection were established. The first scenario is when node $n_{1}$ can establish direct communication with $n_{2}$ and $n_{3}$. The complete information flow model for this scenario is formulated as:

$$
F_{1}=f_{1,2}+f_{1,3}
$$

where $F_{1}$ is the information flow model for the first scenario, $f_{1,2}$ and $f_{1,3}$ are as defined in 3 and 4 respectively. The second information flow scenario is formulated for a situation where $n_{1}$ can only connect directly to $n_{2}$ who then connect with node $n_{3}$. This scenario is formulated as in 8 .

$$
F_{2}=f_{1,2}+f_{2,3}
$$

Similarly, the third scenario given in 9 was formulated when node $n_{1}$ can only connect to $n_{3}$ which then connects to $n_{2}$.

$$
F_{3}=f_{1,3}+f_{3,2}
$$

where $F_{2}$ and $F_{3}$, are the information flow for the second and third scenarios receptively, $f_{2,3}$ and $f_{3,2}$ are as defined in 5 and 6 respectively. To effectively model the overall communications between the three FANET nodes, the following assumptions were made.

- There is no information flow through $f_{2,3}$ and $f_{3,2}$ if $n_{1}$ can establish a connection with $n_{2}$ and $n_{3}$ directly.

- Simultaneous flow of information through $f_{2,3}$ and $f_{3,2}$ cannot exist. 
- Information flow through $f_{2,3}$ or $f_{3,2}$ can only exist if $n_{1}$ can not establish a connection with wither of $n_{2}$ or $n_{3}$,

Base on the above assumptions, the overall information flow model can be formulated as follows:

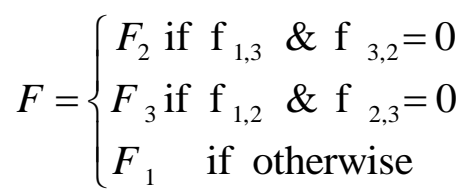

where $F$ denotes the overall communication between the FANET, $f_{1,2}, f_{1,3}, f_{2,3}$ and $f_{3,2}$ are the link connectivity as described above.

\section{EVALUATING FANET NETWORK PERFORMANCE}

In order to minimize the link breakage due to the fast nature of topology change in FANET, the proposed destination path flow model used the flow-based routing technique. Practically, before nodes can exchange information, there should be a path between every user pair node. Irrespective of the speed of the nodes and the topology change, connectivity must be established effectively. The formulations of the path flow models are presented as follows.

\subsection{Definition: destination link formulation}

Considering the network topology $G(N, C)$ given in Figure 2. Four paths which are represented in (3-6) exists. In this case, the intermediate nodes are the hop count. For this topology, only one hop count exists to any destination node. Therefore, nodes can directly connect to one another or through the intermediate nodes depending on the distance between nodes and available capacity of each link. As such, the function to be optimized would consist of maximizing the sum of all path to destination $t$ with respect to the distance between nodes and available capacity of each link. Thus, the multicast transmission model, which is the link capacity optimization objectives can be stated as in (11).

$$
C_{\text {Link }}=\max \sum_{f \in F} \sum_{t \in P_{f}} \sum_{(x, y) \in C} F^{t, f}
$$

where; $\mathrm{C}_{\text {Link }}$ is the multicast link capacity, $\sum_{f \in F}$ indicates all the multicast flow transmitted over the network, $\sum_{t \in P_{f}}$ indicates that a path must exist for every node $\mathrm{t}$ and $\sum_{(x, y) \in C} F^{t, f}$ indicates one of the possible paths for flow $\mathrm{f}$ with destination $\mathrm{t}$. Therefore, the FANET optimization algorithm is presented as follows in Table 2 , The bandwidth parameters in Table 3 were obtained from Netflix standard bandwidth requirement for video streaming while PSO and SAO optimization parameters were obtained from the research paper presented in $[29-31]$.

Table 2. FANET optimization algorithm

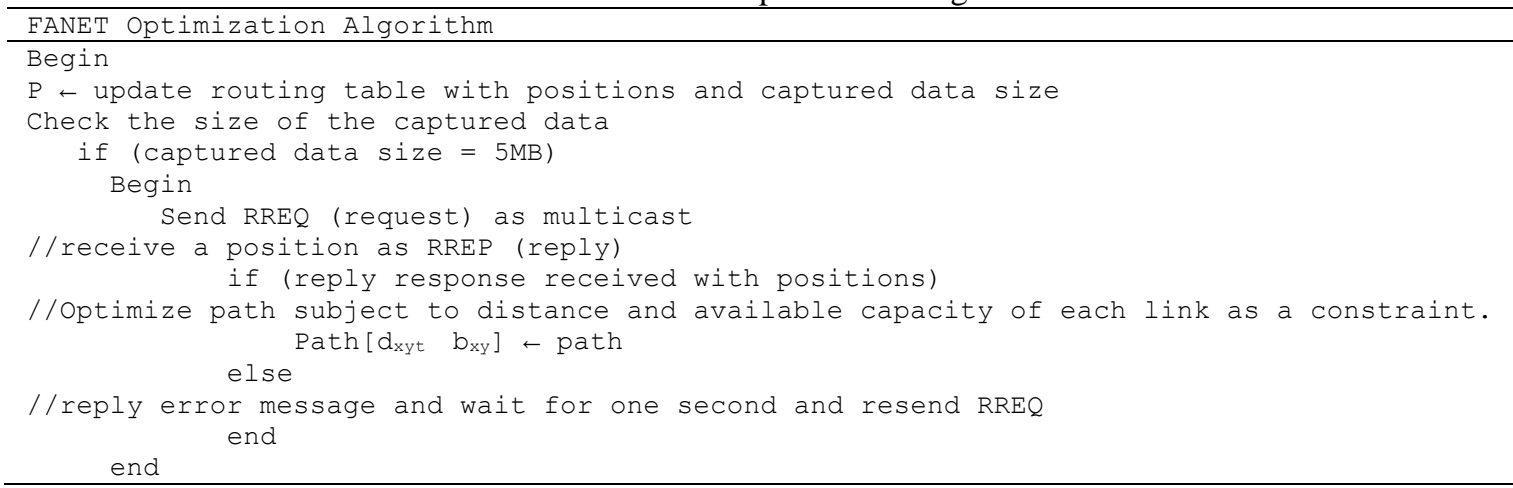


Table 3. Presents the simulation parameters used with the FANET optimization

\begin{tabular}{llll}
\hline S/No. & Parameters & PSO & SAO \\
\hline 1 & Min. Bandwidth (Mbps) & 1.5 & 1.5 \\
2 & Max. Bandwidth (Mbps) & 3.0 & 3.0 \\
3 & Population (Pop) & 50 & 50 \\
4 & FANET Nodes & 3 & 3 \\
5 & Iteration & 100 & 100 \\
\hline
\end{tabular}

\subsubsection{Link connectivity optimization}

The link optimization algorithm is described as follows:

\subsubsection{Particle swarm optimization (PSO)}

PSO is one of the pioneer swarm optimization algorithms which was developed using the principle of flocks of birds and school of fish. The process of PSO usually begins by initializing a set of a randomly generated initial population of solution. Each potential solution is assigned a random velocity with which they are flown into the optimization hyperspace. For the purpose of this paper, the PSO was initialized as a set of information flowing in three-dimensional hyperspace. Each dimension is a representation of a FANET node depicted in Figure 2. The particles keep a record of its own position coordinates in the hyperspace which is associated with the optimum information flow path obtained so far. This particle position coordinate is called the personal best $\left(\mathrm{P}_{\text {best }}\right)$. The overall best particle position is called the global best position is also recorded. This position which represents the overall global information flow is called the global best ( $\left.\mathrm{g}_{\text {best }}\right)$. The mathematical model and details information on PSO implementation can be found in [31].

\subsubsection{Smell agent optimization (SAO)}

Smell agent optimization is a new optimization algorithm developed to mimic the intelligent behavior of an agent trying to identify a smell source. In SOA, the evaporation of smell molecules in the direction of an agent was modeled as sniffing mode. The trailing behavior of the agent towards identifying the smell source is modeled as Trailing mode. Whereas, the intelligent behavior of the agent when a smell trail is lost is modeled into a random mode. In this paper, the smell molecules are initialized as a set of randomly generated information flow through each link. At every stage in the optimization process, the best information flow position is recorded as the agent whose position is required for trailing purpose. Detail information about SAO implementation can be found in $[32,33]$.

\section{EVALUATING FANET NETWORK PERFORMANCE}

This section presents the results and analysis of the paper. Bandwidth minimization process of each algorithm was presented. The optimized bandwidth contribution by each FANET nodes obtained in the form of a cost function is also presented. Three simulation scenarios were considered. The first scenario considered 20 Bandwidth data points for each FANET nodes, the second scenario consider 50 Bandwidth data points for each FANET nodes, whereas, the third scenario considers 100 Bandwidth data points for each FANET nodes as presented in Figures 3-5 and Tables 4-6.
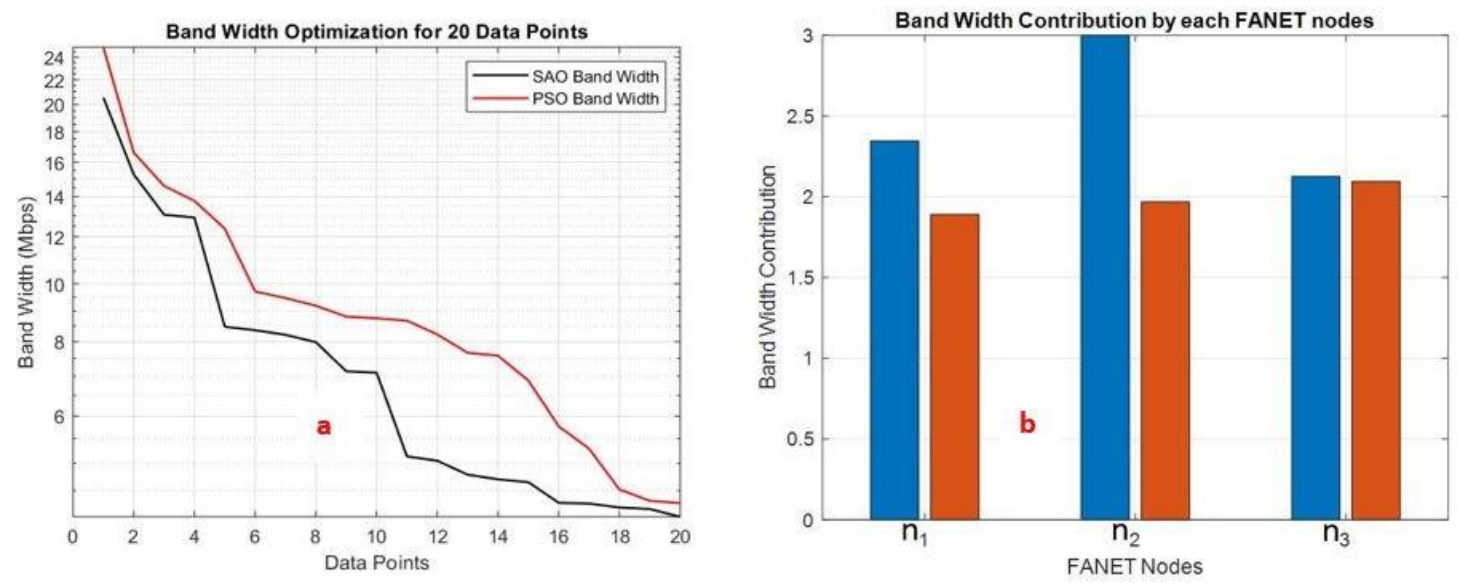

Figure 3. Bandwidth minimization for the first scenario 

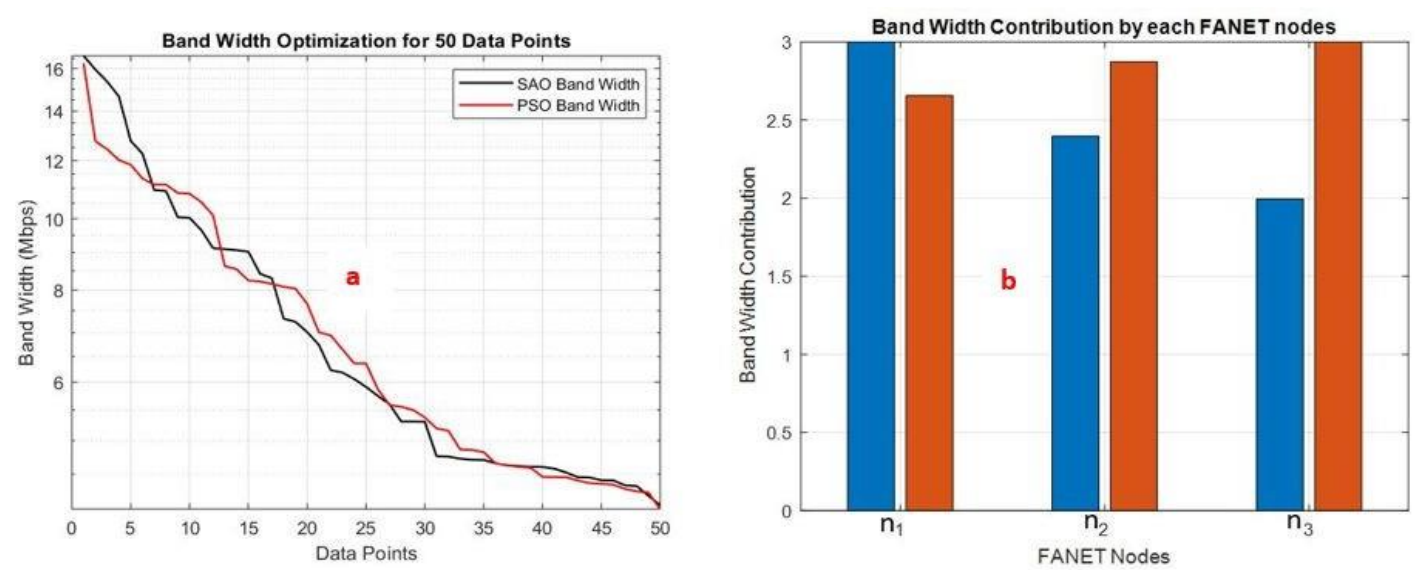

Figure 4. Bandwidth minimization for second scenario
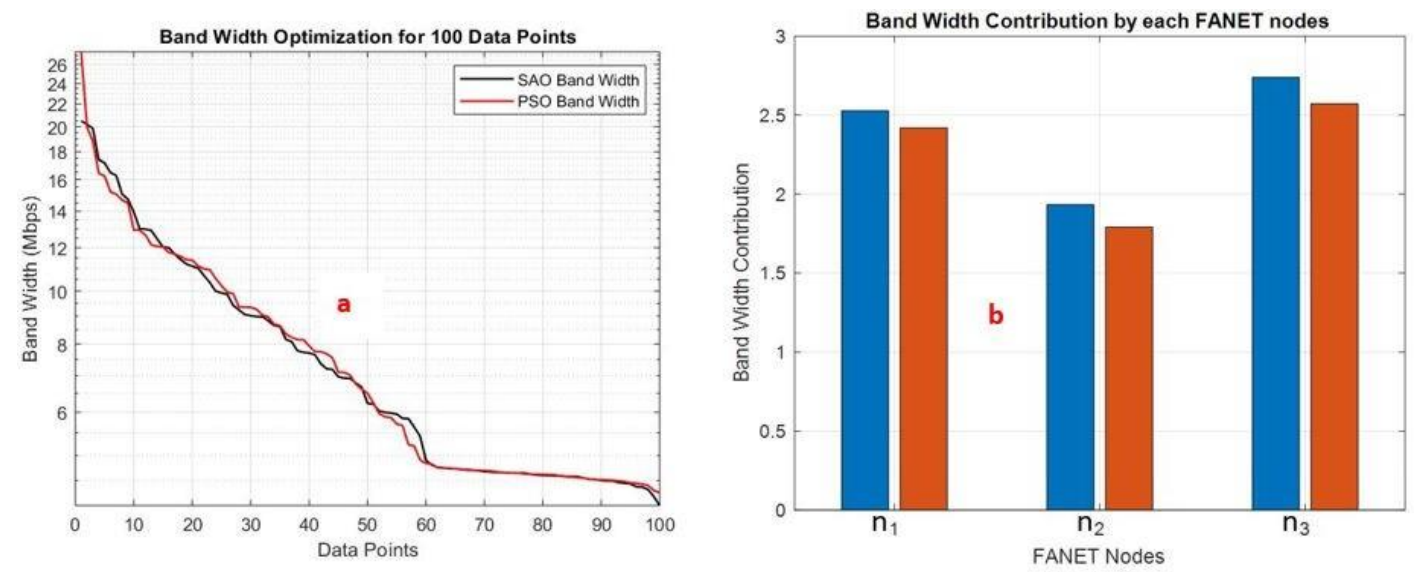

Figure 5. Bandwidth minimization for the third scenario

Table 4. $1^{\text {st }}$ Scenario FANET optimize results

\begin{tabular}{llll}
\hline $\mathrm{S} / \mathrm{n}$ & Algorithm & Cost Function (Mbps) & Average Delay (secs) \\
\hline 1 & PSO & 4.2352 & 0.011044 \\
2 & SAO & 4.0855 & 0.035449 \\
\hline
\end{tabular}

Table 5. $2^{\text {nd }}$ Scenario FANET optimize results

\begin{tabular}{llll}
\hline S/n & Algorithm & Cost Function (Mbps) & Average Delay (secs) \\
\hline 1 & PSO & 4,2130 & 0,041232 \\
2 & SAO & 4,0496 & 0,082887 \\
\hline
\end{tabular}

Table 6. $3^{\text {rd }}$ Scenario FANET optimizeresults

\begin{tabular}{llll}
\hline S/n & Algorithm & Cost Function (Mbps) & Average Delay (secs) \\
\hline 1 & PSO & 4,1617 & 0,096116 \\
2 & SAO & 3,7675 & 0,162613 \\
\hline
\end{tabular}

In all the scenarios considered, both PSO and SAO minimizes the bandwidth required for effective communication between the FANET Nodes. The summary of the results obtained for each scenario is given in the following tables. From Table 4 to Table 6, it can be observed both algorithms optimized the total bandwidth required for effective communication between the FANET nodes. In Table 4, in all the tables, the SAO optimizes the bandwidth costs much better than PSO with a percentage improvement of $10.46 \%$, $4.04 \%$ and $3.66 \%$ with respect to the $1^{s t}, 2^{\text {nd }}$ and $3^{\text {rd }}$ scenarios respectively. In the case of communication delay between the FANET nodes, the PSO has a much better communication delay over SAO with percentage 
improvement of $40.89 \%, 50.26 \%$ and $68.85 \%$ in the first, second and third scenarios respectively. This implies that an optimized destination path flow model improves communication in FANET by preventing closed loop routing and preventing link breakage. Both optimization algorithms used improved communication in FANET. However, using the SAO, bandwidth cost is better while communication delay is better using the PSO as seen in the results.

\section{CONCLUSION}

This paper presents a destination path flow model optimization using particle swarm optimization and smell agent optimization. The interest of this research paper which was to prevent closed loop routing that negatively affects link capacity and efficiency as well as causing link breakage in FANET was largely met. The paper designed and implemented a realistic path model for information dissemination in FANET using optimization algorithms with application to sport and media coverage. Based on this, a routing methodology was proposed. The result achieved showed that the SAO optimizes the bandwidth costs much better than PSO with a percentage improvement of $10.46 \%, 4.04 \%$ and $3.66 \%$ with respect to the $1^{\text {st }}, 2^{\text {nd }}$ and $3^{\text {rd }}$ scenarios respectively. In the case of communication delay between the FANET nodes, the PSO has a much better communication delay over SAO with percentage improvement of $40.89 \%, 50.26 \%$ and $68.85 \%$ in the first, second and third scenarios respectively. Therefore, the objective of the research which was to develop an optimized destination path model was largely Met.

\section{ACKNOWLEDGMENTS}

This research work was sponsored by Tertiary education trust fund (TETFUND) Institution based research (IBR) 2019, under grant no. DAPM/TETFUND/01/12 of Ahmadu Bello University Zaria.

\section{REFERENCES}

[1] Bekmezci I., Sahingoz O. K., and Temel Ş, "Flying ad-hoc networks (FANETs): A survey," Ad-Hoc Networks, vol. 11, no. 3, pp. 1254-1270, 2013.

[2] Bashir O. S., Adewale E.A, Mohammed B.M, Yusuf A.S, "A Specific Routing Protocol for Flying Adhoc Network," TELKOMNIKA Telecommunication Computing Electronics and Control, vol. 16, no. 2, pp. 606-617, 2018.

[3] Oubbati, O. S., Lakas, A., Zhou, F., Güneş, M., Yagoubi, M. B, “A survey on position-based routing protocols for Flying Ad hoc Networks (FANETs),” Vehicular Communications, vol. 10, pp. 29-56, 2017.

[4] Gankhuyag G., Shrestha A. P., and Yoo S.-J, "Robust and Reliable Predictive Routing Strategy for Flying Ad-Hoc Networks," IEEE Access, vol. 5, no. 1, pp. 643-654, 2017.

[5] Seilendria A. H., Carlos T. C., Juan-Carlos C., Yusheng J., Enrique H., Pietro M, "3D Simulation Modeling of UAV-to-Car Communications," IEEE Access, vol. 7, no. 1, pp. 8808-8823, 2019.

[6] Li J., Zhou Y., and Lamont L, "Communication Architectures and Protocols for Networking Unmanned Aerial Vehicles," Lobecom Workshop - Wireless Networking and Control for Unmanned Autonomous Vehicles, vol. 1, no. 1, pp. 1415-1420, 2013.

[7] Pahlavan K., Levesque A.H, "Wireless Information Networks," 2nd ed. John Wiley \& Sons, publishers Inc, 2015.

[8] Zeng Y., Zhang R., Lim T. J, "Wireless communications with unmanned aerial vehicles: opportunities and challenges," IEEE Communications Magazine, vol. 54, no. 5, pp. 36-42, 2016.

[9] Orhan D. Abdullah K. Alice E. S, "Connectivity management in mobile ad hoc networks using particle swarm optimization,” Ad Hoc Networks Journal, Elsevier, pp. 1312-1316, 2011.

[10] Wei, Z., Wu, H., Feng, Z., Chang, S, “Capacity of UAV Relaying Networks,” IEEE Access, vol. 1, no. 1, 2019.

[11] Singh K., Verma A. K, "Experimental Analysis of AODV, DSDV and OLSR Routing Protocol for Flying Adhoc Networks (FANETs)," IEEE Transactions, vol. 1, no. 1, pp. 1-4, 2015.

[12] Sahingoz O. K, "Networking models in flying Ad-hoc networks (FANETs): Concepts and challenges," Journal of Intelligent \& Robotic Systems, vol. 74, no. 1-2, pp. 513-527, 2014.

[13] Tareque H., Hossain S., and Mohammed A, "On the routing in Flying Ad-Hoc Networks," Proceedings of the Federated Conference on Computer Science and Information Systems (FedCSIS), vol. 5, pp. 1-9, 2015.

[14] Rosati S., Kruzelecki K., Heitz G., Floreano D., and Rimoldi B, "Dynamic Routing for Flying Ad-Hoc Networks," IEEE Transactions on Vehicular Technology, vol. 63, no. 3, pp. 1690-1700, 2016.

[15] Wajiya Z., Bilal M. K, "Flying Ad Hoc Networks: Technological and Social Implications," IEEE Technology and Society Magazine, pp. 67-74, 2016.

[16] Bekmezci I, Ermis M., Kaplan S, “Connected Multi UAV Task Planning for Flying Ad Hoc Networks,” IEEE International Black Sea Conference on Communications and Networking (BlackSeaCom), Odessa, pp. 28-32, 2014.

[17] Jianping W., Eseosa O., Parimala T., Ruppa K. T, "HOPNET: A hybrid ant colony optimization routing algorithm for mobile ad hoc network," Ad Hoc Networks: Elsevier Journal, pp. 690-705, 2009.

[18] Shubhajeet C., Swagatam D, "Ant colony optimization based enhanced dynamic source routing algorithm for mobile Ad-hoc network,” Information Sciences: Elsevier Journal, pp. 67-90. 2015. 
[19] Salim B., Abdelhamid M., Sherali Z, "Bio-Inspired Routing Algorithms Survey for Vehicular Ad-hoc Networks," IEEE Communications Surveys \& Tutorials, pp. 1-25, 2014.

[20] Hajlaoui, R., Guyennet, H., Moulahi, T, "A Survey on Heuristic-Based Routing Methods in Vehicular Ad-Hoc Network: Technical Challenges and Future Trends," IEEE Sensors Journal, vol. 16, no. 17, pp. 6782-6792, 2016.

[21] Sara R., Abdellah I, "A multi-objective optimization system for mobile gateways selection in vehicular Ad-Hoc networks," Computers and Electrical Engineering: Elsevier Journal, vol. 73, pp. 289-303, 2019.

[22] Zahid D, Pingzhi F., Sangsha F, "On the Connectivity of Vehicular Ad hoc Network Under Various Mobility Scenarios," IEEE Access Journal, vol. 5, pp. 22559-22566, 2017.

[23] Kumar S., Ahmed S.H, Qasim U., Khan Z.A, Amjad N., Azeem M.Q., Ali A., Ashraf M.J., Javaid N, “Analyzing Link and Path Availability of Routing Protocols in Vehicular Ad hoc Networks," Journal of Basic and Applied Science Research, vol. 4, no 2, pp. 189-206, 2014.

[24] Tariq U., Muhammed K.A., Ehasan U. M., Muhammed A, “A Dual Ring Model for VANET under Heterogeneous Traffic Flow,” Wireless Pers Commun: Springer Journal, 2017.

[25] Muhammad A. K., Ijaz M. Q., Fahimullah K, "A Hybrid Communication Scheme for Efficient and Low-Cost Deployment of Future Flying Ad-Hoc Network (FANET)," Drones Journal: mdpi, vol. 3, no. 16, pp. 1-20, 2019.

[26] Antonio G., Maria-Dolores C, "Flying Ad Hoc Networks," A New Domain for Network Communications. Sensors Journal, vol. 18, pp. 1-23, 2018.

[27] Farhan A., Ali R., Muhammad F. K., Muazzam M., Irfan M., "Seungmin R. Energy-Aware Cluster-Based Routing in Flying Ad-Hoc Networks," Sensors Journal, vol. 18, pp. 1-16, 2018.

[28] Armir B., Carlos T.C., Juan-Carlos C., Pietro M., Claudio E. P., Daniele R, "Flying Ad-hoc Network Application Scenarios and Mobility Models," International Journal of Distributed Sensor Networks, vol. 13, no. 10, pp. 1-17, 2017.

[29] Khawaja B. A., Tarar M. A., Tauqeer T., Amir F., Mustaqim M, "A $1 \times 2$ triple-band printed antenna array for use in next-generation flying AD-HOC networks (FANETs)," Microwave and Optical Technology Letters, vol. 58, no. 3, pp. 606-610, 2016.

[30] Whitehouse, K., Karlof, C., Culler, D, "A practical evaluation of radio signal strength for ranging-based localization, "ACM SIGMOBILE Mobile Computing and Communications Review," vol. 11, no. 1, pp. 41-52, 2007.

[31] Awad, M. K., El-Shafei, M., Dimitriou, T., Rafique, Y., Baidas, M., and Alhusaini, A, "Power-efficient routing for SDN with discrete link rates and size-limited flow tables: A tree-based particle swarm optimization approach," International Journal of Network Management, 2017.

[32] A.T Salawudeen, M.B Mu'azu, Y. Shaaban and E. A. Adedokun, "On the Development of Novel Smell Agent Optimization for Optimization Problems," $2^{\text {nd }}$ international conference on Information Communication Technology and Its Application, pp. 287-297, 2018.

[33] A.T Salawudeen, M.B Mu'azu, Y. Shaaban and E. A. Adedokun, "From Smell Phenomenon to smell Agent Optimization: A feasibility study, Proceedings," of the international conference on global and emerging trends (ICGET), pp. 78-85, 2018.

\section{BIOGRAPHIES OF AUTHORS}

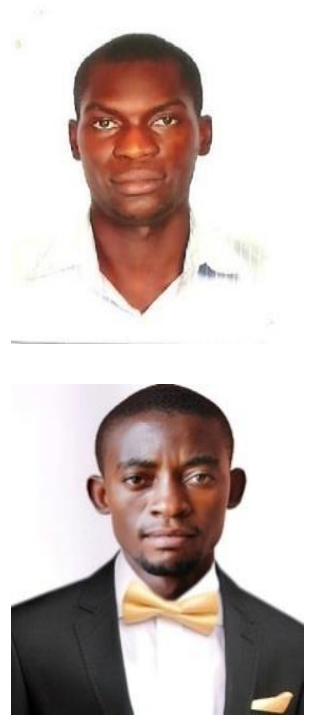

Bashir Olaniyi Sadiq is a Lecturer of the Department of Computer Engineering, Ahmadu Bello University Zaria. He obtained his B. Eng., MSc and Ph.D. degrees in Computer Engineering from Ahmadu Bello University Zaria. He is a member of IEEE, IAENG, NSE, and Council for the Regulation of Engineering in Nigeria. His areas of Expertise are Networks and Image Processing.

Ahmed Tijani Salawudeen is a Lecturer of the Department of Computer Engineering, Ahmadu Bello University Zaria. He obtained his B. Eng., MSc and Ph.D. degrees in Control Engineering from Ahmadu Bello University Zaria. He is a member of IAENG, IEEE, INSTICC, NSE and Council for the Regulation of Engineering in Nigeria. His areas of Expertise are Operations research and Control Systems. 